\title{
Role of conventional salvage multiple-drug chemotherapy in relapsed and refractory aggressive non-Hodgkin lymphomas
}

\author{
PAOLO G. GOBBI，LARA VILLANO，DONATELLA POZZOLI and MANUELA BERGONZI
}

Medicina Interna e Gastroenterologia, Università di Pavia, Fondazione IRCCS Policlinico S. Matteo, Pavia, Italy

Received February 4, 2010; Accepted April 13, 2010

DOI: 10.3892/ol_00000119

\begin{abstract}
Autologous stem cell transplantation is the standard care for patients with relapsed or refractory aggressive nonHodgkin lymphomas. Of the patients who are sensitive to second-line chemotherapy, approximately $40-50 \%$ are likely to be cured using this approach. The optimal salvage regimen for pre-transplant debulking is controversial and these second-line chemotherapies are particularly important for patients who cannot undergo transplantation for various reasons including age, comorbidity and insufficient stem cell collection. Numerous reports regarding this topic are available. This study evaluated reports published in the last 5 years, focusing on conventional multiple-drug second-line chemotherapies (with or without rituximab), and disregarding single-agent investigational phase-II trials. Results are encouraging, particularly when considering that the more recent and less toxic combinations appear to be equivalent to or even more favourable than previous, more aggressive approaches. Previous results obtained using a combination of mitoxantrone, carboplatin, cytarabine and methylprednisolone, are further updated and included in this study. In conclusion, the most effective conventional chemotherapy currently available for patients with relapsed or refractory nonHodgkin lymphomas obtains complete remission rates of up to 50-70\%; the achievement of a complete remission is the most important factor associated with a better outcome. Although the addition of rituximab is beneficial and safe, it is more effective in patients who have previously not been exposed to this monoclonal antibody. The addition of cycles of salvage chemotherapy to those strictly required for mobilization of peripheral blood stem cells ultimately improves the response rate.
\end{abstract}

\section{Introduction}

Histologically aggressive types of non-Hodgkin lymphomas are considered to be among the disorders most sensitive to chemo-

Correspondence to: Professor Paolo G. Gobbi, Clinica Medica I, Università di Pavia, Fondazione IRCCS Policlinico S. Matteo, Piazzale Golgi no. 2, 27100 Pavia, Italy

E-mail: gobbipg@smatteo.pv.it

Key words: non-Hodgkin lymphoma, relapse, refractoriness, salvage chemotherapy therapy and radiotherapy. However, a substantial fraction of patients (40-50\%) with such disorders fail to achieve complete remission on first-line treatment $(1,2)$. Approximately $10 \%$ of the subjects develop progressive disease during their initial therapy, and must be considered as truly refractory patients. Moreover, approximately $50 \%$ of complete responders eventually relapse. The prognosis of these patients is generally poor. The improvement of transplantation techniques has enhanced the outlook for nearly $50 \%$ of refractory or relapsed patients using high-dose chemotherapy (HDC), followed by autologous (ASCT) or allogeneic (Allo-SCT) stem-cell transplantation (3). Nevertheless, the response to transplantation procedures depends on a number of factors such as presentation of the disease, type and number of previous therapies, prognostic factors, response to debulking chemotherapy regimens administered before HDC and the type of pre-transplant conditioning regimen used. Chemosensitivity to the disease prior to bone marrow transplantation has been shown, in particular, to be a significant discriminator of the final outcome, with complete disappearance of symptoms. According to certain authors (4), patients who do not respond to second-line (salvage) chemotherapy should not be offered ASCT, but should be candidates for alternative or experimental therapies. However, if their response to salvage therapy is excluded, HDC is not a viable treatment option for all refractory or relapsed patients. There are various reasons for this, including comorbidity, frailty, advanced age and risk of complications. Therefore, these patients must be maintained in an acceptable clinical condition and their life prolonged with conventional chemotherapy. Chemotherapy protocols have been designed for pre-transplant cytoreduction and complete remissions have been achieved in a significant number of cases.

This study provides a brief overview of the conventional multiple-drug regimens that have recently been designed and tested to improve the outlook of patients with relapsed or refractory aggressive non-Hodgkin lymphoma.

\section{Materials and methods}

Numerous studies, as well as bibliographic references, have reported on the treatment of relapsed or refractory aggressive non-Hodgkin lymphomas. In this study, the field of interest was restricted to clinical studies published in the last 5 years. These studies included at least 10 patients who either had histologically aggressive types of non-Hodgkin lymphomas or 
findings regarding those patients with aggressive lymphomas were able to be extrapolated. Phase II studies with single-drug therapy (with cytostatic or biological agents) were excluded, even in the case of promising results, since results from any comparison and possible implementation with conventional drugs were inconclusive. Studies employing radio-immunoconjugates were also excluded since these drugs are limited to the investigational setting, show marked myeloablative effects, and their administration remains confined to selected, well-experienced centers.

The parameters selected to evaluate and compare the effectiveness of the salvage regimens and the value of the reports were: number of patients evaluated, median or range (or both) of the number of chemotherapy lines previously administered, overall response rate (ORR, i.e., the cumulative percentage of partial and complete remissions), complete remission rate [CRR, which is the best prognostic discriminant, particularly in relapsed or refractory patients (5)], number of cycles administered and power of peripheral blood stem cell (PBSC) mobilization ( + , sufficient for an autologous stem cell transplant but $<6 \times 10^{6} / \mathrm{kg} \mathrm{CD} 34^{+}$cells, and,$++>6 \times 10^{6} / \mathrm{kg} \mathrm{CD} 34^{+}$cells).

A previous study reported on a favourable response rate to an outpatient regimen, i.e., the MJMA schedule using the combination of mitoxantrone, carboplatin, cytarabine and methylprednisolone (6). In this study, the results of the previous series of patients are updated. Subsequently, 4 cases with Hodgkin lymphoma were excluded and 3 further patients with diffuse large B-cell lymphoma were included. Two of the latter patients had relapsed following R-CHOP and 1 was refractory to R-CHOP. The number of patients with aggressive non-Hodgkin lymphomas treated with the salvage MJMA regimen was 27 . The outcome of the 27 patients is included.

The MJMA chemotherapy was a 3-day, shortened and intensified variant, with a 3-week interval of the known 5-day MiCMA regimen that is delivered at 4 -week intervals $(7,8)$. The modifications were made to increase the dose size of carboplatin and the dose intensity of all the drugs, while maintaining the feasibility of the treatment in an outpatient setting and without modifying the cumulative doses administered. The schedule revised in this way consisted of mitoxantrone $10 \mathrm{mg} /$ $\mathrm{m}^{2}$ intravenously (i.v.) on Day 1, carboplatin $200 \mathrm{mg} / \mathrm{m}^{2}$ daily i.v. on Days 1 and 2, cytarabine $2000 \mathrm{mg} / \mathrm{m}^{2}$ i.v. on Day 3 and methylprednisolone $500 \mathrm{mg} / \mathrm{m}^{2}$ daily i.v. on Days $1-3$. Treatment was repeated at 21-day intervals for a total of 4-6 cycles. The theoretical mean dose intensity of all the drugs was increased by $25 \%$ and the dose size of carboplatin was doubled.

\section{Results}

Table I shows the data relating to 35 distinct studies and 1,558 patients. CRR, one of the most reliable indicators of effectiveness, ranges from 14 to $79 \%$, while ORR varies from 28 to $94 \%$.

In our updated series of 27 non-Hodgkin patients included in Table I (17 with relapsed disease and 10 refractory prior to treatment), 18 (64\%) achieved complete remission (12 relapsed, 6 refractory) and 8 (30\%) obtained only a partial remission (4 relapsed, 4 refractory). ORR was $94 \%$ with only 1 patient, who had been refractory to four previous chemotherapy regimens, not responding to MJMA.
The 27 patients experienced severe hematological toxicity (grades 3 and 4 of the WHO scale). Severe neutropenia was observed in all 22 patients and severe thrombocytopenia in 19 of 27 patients. Anemia, with a hemoglobin concentration $<8 \mathrm{~g} / \mathrm{dl}$ (WHO grade 2) was noted in 6 patients. Of the 27 patients, 18 (aged 27-64) for whom consolidation therapy with HDC and subsequent peripheral blood stem cell (PBSC) rescue was judged appropriate and feasible, underwent marrow stimulation with granulocyte colony-stimulating factor after one of the first three cycles $(300 \mu \mathrm{g}$ every $12 \mathrm{~h}$ from Day 5 until completion of the leukapheresis procedures). Mobilization of PBSC was successful in these patients, with a mean yield of $\mathrm{CD} 4^{+}$cells of $10.45 \times 10^{6} / \mathrm{kg}$ per patient (range $3.70-24.88$ ). A single leukapheresis was sufficient in 15 of 18 patients, while two leukaphereses were necessary in the remaining 3 .

\section{Discussion}

An effective salvage therapy for non-Hodgkin lymphoma should have low hematological and non-hematological toxicity and good power to mobilize PBSC. Additionally, and most importantly, this therapy should exhibit sufficiently strong cytoreductive activity in order for an adequate number of cycles to be administered, with favourable results, in patients who cannot undergo ASCT.

As noted in Table I, none of the presently available regimens fulfills these prerequisites. Chemoresistance poses the main difficulty, as demonstrated by patients whose disease progressed during or soon after front-line therapies and by patients who relapsed early following such therapies. Findings regarding salvage therapy should refer not only to the number of refractory cases presented, but should also list outcomes separately for refractory and relapsed patients. However, studies rarely report on such outcomes.

It must also be considered that patients with refractory or relapsed lymphomas normally show a reduction in tolerance of salvage chemotherapies compared to front-line regimens due to iatrogenic myelosuppression, variable degrees of marrow infiltration by lymphoma, the onset of newly acquired metabolic abnormalities or worsening of those underlying the lymphoma and a number of possible clinical complications. Consequently, drug doses often have to be reduced. Nevertheless, administration of maximum tolerated doses is necessary in order to achieve the maximum response. Finally, randomized trials are difficult to organize in this setting of patients because of the number of clinical variables that play important clinical roles and, theoretically, require many stratifications, such as histology, tumor bulk, response to and number of previous treatments, symptoms and presence of prognostic factors prior to salvage therapy.

The majority of studies noted that a number of the treated patients received HDC, followed by ASCT or Allo-SCT, making it difficult to compare the intrinsic long-term efficacy of the conventional salvage chemotherapy. For this reason, final response was considered, but not survival parameters. However, since HDC is not a viable treatment option for all refractory or relapsed patients due to factors, including comorbidity, frailty, advanced age and risk of infections, these patients must be maintained in an acceptable clinical condition and their life should be prolonged with conventional chemotherapy. 
Table I. Overall response and complete remission rate obtained by the salvage regimens tested in recent years. ${ }^{\mathrm{b}}$

\begin{tabular}{|c|c|c|c|c|c|c|}
\hline Regimen (reference) & $\begin{array}{l}\text { No. of } \\
\text { patients }\end{array}$ & $\begin{array}{l}\text { No. of previous } \\
\text { regimens }\end{array}$ & $\begin{array}{l}\text { ORR } \\
(\%)\end{array}$ & $\begin{array}{l}\text { CRR } \\
(\%)\end{array}$ & $\begin{array}{l}\text { No. of } \\
\text { cycles }\end{array}$ & $\begin{array}{l}\text { Mobilization } \\
\text { ability }\end{array}$ \\
\hline IIVP (IFO, IDA, VP) (9) & 49 & $1-2$ & 76 & 33 & 3 & n.g. \\
\hline DICE (Dexa, IFO, CDDP, VP) (10) & 35 & 1 & 74 & 31 & 4 & n.g. \\
\hline IEV (IFO, EPI, VP) (11) & 34 & $1-2$ & 77 & 64 & 3 & ++ \\
\hline IEV-R (IFO, EPI, VP, Rit) (12) & 16 & $1.5(1-3)$ & 69 & 38 & 2 & ++ \\
\hline IEV (IFO, EPI, VP) (12) & 59 & $1.5(1-3)$ & 41 & 19 & 2 & ++ \\
\hline IEV (IFO, EPI, VP) (13) & 41 & $1-4$ & 66 & 27 & 2 & ++ \\
\hline ICE (IFO, CBDCA, VP) (14) & 75 & $1.5(1-4)$ & 89 & 29 & 2.8 & ++ \\
\hline ICE (IFO, CBDCA, VP) (15) & 45 & $2(1-5)$ & 45 & 17 & 3 & ++ \\
\hline R-ICE (Rit, IFO, CBDCA, VP) (16) & 21 & $\geq 2$ (DHAP) & 52 & 14 & 3 & n.g. \\
\hline R-ICE (Rit, IFO, CBDCA, VP) (17) & 36 & 1 & 78 & 53 & 3 & ++ \\
\hline IVAD (IFO, VP, ARA-C, Dexa) (18) & 59 & 1 & 67 & 19 & $2-3$ & ++ \\
\hline GVP (GEM, VRL, Pred) (19) & 15 & 3 & 53 & 33 & 3.2 & n.g. \\
\hline GV (GEM, VRL) (20) & 22 & 1 & 50 & 14 & $3-6$ & n.g. \\
\hline GP (GEM, CDDP) (21) & 30 & 1 & 53 & 53 & 3 & n.g. \\
\hline GDP (GEM, Dexa, CDDP) (22) & 51 & 1 & 49 & 16 & 2 & ++ \\
\hline GDP (GEM, Dexa, CDDP) (23) & 24 & 1 & 58 & 21 & 3 & + \\
\hline ViGePP (VRL, GEM, PCZ, Pred) (24) & 66 & $1-3$ & 46 & 23 & 5 & n.g. \\
\hline R-GIFOX (Rit, GEM, IFO, OX) (25) & 13 & $2(1-4)$ & 77 & 50 & 3 & + \\
\hline GEMOX-R (Rit, GEM, OX) (26) & 32 & 1.7 & 43 & 34 & 4 & n.g. \\
\hline GEM-P (GEM, CDDP, m-Pred) (27) & 39 & $2(1-5)$ & 39 & 79 & 21 & ++ \\
\hline GEM-P (GEM, CDDP, m-Pred, \pm Rit) (28) & 39 & $1-3$ & 59 & 28 & 2 & n.g. \\
\hline R-GEM (Rit, GEM) (29) & 33 & $\geq 2$ & 48 & 24 & 2 & n.g. \\
\hline DHAP (Dexa, ARA-C, CDDP) (30) & 28 & 1 & 68 & 28 & 2 & ++ \\
\hline DHAP (Dexa, ARA-C, CDDP) (31) & 53 & $1-3$ & 62 & 37 & $3-4$ & n.g. \\
\hline DHAP (Dexa, ARA-C, CDDP) (32) & 57 & 1 & 72 & 9 & 2 & a \\
\hline $\begin{array}{l}\text { DHAP-VIM-DHAP } \\
\text { (Dexa, ARA-C, CDDP, VP, IFO, MTX) (33) }\end{array}$ & 101 & 1 & 54 & n.g. & 3 & n.g. \\
\hline $\begin{array}{l}\text { R-DHAP-VIM-DHAP } \\
\text { (Rit, Dexa, ARA-C, CDDP, VP, IFO, MTX) (33) }\end{array}$ & 101 & 1 & 77 & n.g. & 3 & n.g. \\
\hline ESHAP (VP, m-Pred, ARA-C, CDDP) (34) & 22 & $1-3$ & 63 & 27 & $2-4$ & ++ \\
\hline R-ESHA (Rit, VP, m-Pred, ARA-C, CDDP) (35) & 94 & $1\left(\mathrm{Rit}^{+}\right)$ & 67 & 37 & 3 & \\
\hline R-ESHAP (Rit, VP, m-Pred, ARA-C, CDDP) (35) & 69 & 1 ( (Rit) $)$ & 81 & 56 & 3 & ++ \\
\hline MiCMA (Mito, CBDCA, ARA-C, m-Pred) (36) & 85 & $1-4$ & 70 & 26 & $2-3$ & ++ \\
\hline MJMA (Mito, CBDCA, ARA-C, m-Pred) (37) & 31 & $1-3$ & 72 & 45 & 5 & n.g. \\
\hline 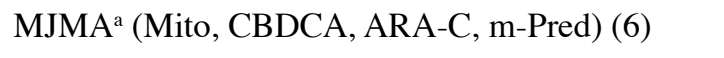 & 27 & 1.5 & 94 & 64 & 5 & ++ \\
\hline $\begin{array}{l}\text { Dexa-BEAM } \\
\text { (Dexa, BCNU, VP, ARA-C, MPH) (38) }\end{array}$ & 29 & 1 & 28 & 3 & $1-2$ & + \\
\hline R-EPOCH (Rit, DOX, VP, VCR, CTX, Pred) (39) & 50 & 1.7 & 68 & 28 & 4 & n.g. \\
\hline $\begin{array}{l}\text { R-H-CVAD/ R-AM } \\
\text { (Rit, CTX, DOX, VCR, Dexa/ARA-c, MTX) (40) }\end{array}$ & 29 & $1(1-5)$ & 93 & 45 & 5 & n.g. \\
\hline CMC (2-CdA, Mito, CTX) (41) & 33 & $1-2$ & 58 & 21 & 4 & n.g. \\
\hline CEMP (CDDP, VP, Mito, Pred) (42) & 47 & 1 & 34 & 23 & 5 & n.g. \\
\hline
\end{tabular}

ORR, overall response rate; $\mathrm{CRR}$, complete remission rate; ARA-C, cytarabine; BCNU, carmustine; CBDCA, carboplatin; 2-CdA, cladribine; CCNU, lomustine; CDDP, cisplatin; CTX, cyclophosphamide; Dexa, dexamethasone; DOX, doxorubicin; EPI; epidoxorubicin; IFO; ifosfamide; MPH, melphalan; m-Pred, methylprednisolone; Mito, mitoxantrone; MTX, methotrexate; PCZ, procarbazine; Pred, prednisone; Rit, rituximab; VCR, vincristine; VP, etoposide; VRL, vinorelbine; n.g., not given;,$+ \leq 6 \times 10^{10} / \mathrm{kg} \mathrm{CD} 34^{+} ;++,>6 \times 10^{10} / \mathrm{kg} \mathrm{CD}^{2} 4^{+}$. ${ }^{\mathrm{a} D a t a}$ of ref. 6 are

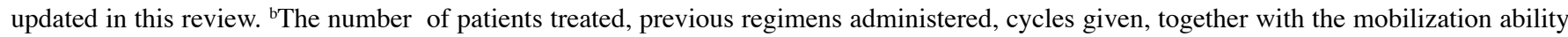
of PBSC, are also reported. 
Three main groups of regimens are identified according to their pivotal drugs or combination of drugs. The first group comprises ifosfamide-based regimens (9-18) that produce complete remission rates ranging from 14 to $64 \%$. The toxicity of these regimes is mainly hematological and they generally allow for the adequate collection of PBSC in the majority of patients. The second group is formed by the gemcitabinecontaining regimens, frequently associated with vinorelbine and/or platinum compounds (19-29). These regimens produce complete remission rates ranging from 21 to $43 \%$, and are generally less toxic to bone marrow. Largely due to their lower toxicity, regimes of the second group are often employed in frail cases. Thus, their ability to mobilize PBSC has been tested in fewer studies. However, in those series in which this aspect was explored, the regimens showed favourable mobilization power. The third group comprises regimens containing cytarabine and platinum compounds (6,30-36, La Sala A, et al, Riassunti del $35^{\circ}$ Congr. SIE, Edimes, Pavia, abs. 297, 1995). The regimes produce complete remission rates ranging from 3 to $64 \%$, have marked hematological toxicity and considerable non-hematological toxicity, but are able to mobilize PBSC.

An additional issue is the role of rituximab in combination with conventional chemotherapy in the salvage setting. As shown by the studies reported in Table I $(12,16,17,25,26$, $28,29,33,35,37-39)$, the addition of rituximab to salvage chemotherapy improves the response rate, without decreasing the mobilization and collection of PBSC. Moreover, the original reports provide evidence of the lack of significant toxicity due to the addition of rituximab to salvage chemotherapy. However, in a number of investigations the majority of patients did not previously receive treatment with rituximab. Thus, the conclusions drawn by Martin et al (35), appear to suggest that the impact of rituximab in the salvage setting is limited. The authors concluded that prior exposure to rituximab is an adverse prognostic determinant for the efficacy of the same monoclonal antibody on overall and progression-free survival of relapsed or refractory patients. Martin et al hypothesized that the refractoriness of the disease observed in patients who receive rituximab during induction therapy questions the role of HDC and ASCT in this particular setting. Subsequently, the issue regarding resistance to rituximab in $\mathrm{CD} 20^{+}$nonHodgkin lymphomas, remains to be elucidated (42). The serum concentration of the monoclonal antibody, its catabolic rate, surface expression of CD20 receptors, altered intracellular signaling, altered complement functions and defective cell-mediated immunity are potential factors involved in the resistance to rituximab.

Notably, in a limited series of patients refractory to DHAP, which can be considered a strong second-line chemotherapy, the ORR to R-ICE (16) was 52\%, with $14 \%$ of the patients achieving a complete response. The results of third-line conventional chemotherapy are usually poor. Consequently, patients with an inadequate response to second-line chemotherapy are considered to have a poor recovery rate with subsequent salvage regimens. The novel use of rituximab in the patients treated by Simpson et al (16) explains the promising results obtained with R-ICE.

A further, minor bias affecting the comparability of treatments in the series of relapsed/refractory non-Hodgkin lymphomas is the different number of cycles actually administered. Centers strongly oriented towards ASCT often administer only 2 or 3 cycles in order to allow for the optimal collection of PBSC and early performance of the transplant. In these cases, the clinical response to conventional cytoreductive therapy is evaluated after only 2 to 3 cycles of chemotherapy. In studies that include larger numbers of elderly or otherwise frail patients, who are not eligible candidates for ASCT, more cycles are administered, which may enable better exploitation of the intrinsic anti-lymphoma effectiveness of the salvage regimens. This discrepancy may explain the different results obtained with the same mitoxantrone-carboplatincytarabine-methylprednisolone combination of drugs (MJMA and MiCMA stand for the same drugs, since ' $J$ ' in MJMA refers to one of the first abbreviations of carboplatin, JM-8, represented by the ' $C$ ' in MiCMA). Sica et al (7), La Barbera et al (8) and Sorà et al (36) administered only 2 or 3 cycles of the MJMA regimen and patients received HDC and ASCT soon after harvesting PBSC. Similar to our study, La Sala et al (Riassunti del $35^{\circ}$ Congr. SIE, Edimes, Pavia, abs. 297, 1995) administered 5 or 6 cycles, followed by 2-4 cycles which were then used for PBSC mobilization and harvesting. In addition, the doubled dose size of carboplatin and the dose intensification of all the drugs administered to our patients may explain the higher response rate of our series compared to that of La Sala et al, the number of cycles administered being equal.

In conclusion, with the most effective conventional chemotherapy currently available for relapsed or refractory non-Hodgkin lymphoma, up to $50-70 \%$ of patients obtain complete remission. This is of considerable relevance, given that the achievement of complete remission is the most important factor associated with better survival. The addition of rituximab is useful and safe, although likely more effective in patients not previously exposed to the drug. The addition of more cycles of salvage chemotherapy to those strictly required for the mobilization of PBSC improves the response rate.

\section{Acknowledgements}

The study was supported in part by grants from the Ministero dell'Università e della Ricerca Scientifica e Tecnologica, Roma, the Fondazione IRCCS Policlinico S. Matteo, Pavia, and the Ferrata-Storti Foundation, Pavia, Italy.

\section{References}

1. Fisher RI, Gaynor ER, Dahlberg S, et al: Comparison of a standard regimen (CHOP) with three intensive chemotherapy regimens for advanced non-Hodgkin's lymphoma. N Engl J Med 328: 1002-1006, 1993.

2. Coiffier B, Lepage E, Briere J, et al: CHOP chemotherapy plus rituximab compared with $\mathrm{CHOP}$ alone in elderly patients with diffuse large B-cell lymphoma N Engl J Med 346: 235-242, 2002.

3. Philip T, Guglielmi C, Hagenbeek A, et al: Autologous bone marrow transplantation as compared with salvage chemotherapy in relapses of chemosensitive non-Hodgkin's lymphoma. N Engl J Med 333: 1540-1545, 1995.

4. Ardeshna KM, Kakouros N, Qian W, et al: Conventional second-line salvage chemotherapy regimens are not warranted in patients with malignant lymphomas who have progressive disease after first-line salvage therapy regimens. $\mathrm{Br} \mathrm{J}$ Haematol 130: 363-372, 2005. 
5. Casasnovas RO, Haioun C, Dumontet C, et al: Phase II study of 3-hour infusion of high dose paclitaxel in refractory and relapsed aggressive non-Hodgkin's lymphomas. Groupe d'Etude des Lymphomes de l'Adulte. Haematologica 85: 502-507, 2000.

6. Gobbi PG, Valentino F, Lambelet P, et al: Shortened and intensified MJMA: an effective salvage therapy for relapsed and refractory lymphomas and a strong mobilizer of PBSCs. Bone Marrow Transplant 44: 19-25, 2009.

7. Sica S, Di Mario A, Salutari P, et al: Sequential peripheral blood progenitor cell transplantation after mobilization with salvage chemotherapy and G-CSF in patients with resistant lymphoma. Am J Hematol 46: 18-23, 1994.

8. La Barbera EO, Chiusolo P, Laurenti L, et al: MiCMA: an alternative treatment for refractory or recurrent Hodgkin's disease. Ann Oncol 11: 867-871, 2000.

9. Oyan B, Koc Y, Ozdemir E, et al: Ifosfamide, idarubicin and etoposide in relapsed/refractory Hodgkin disease and nonHodgkin lymphomas: a salvage regimen with high response rates before autologous stem cell transplantation. Biol Blood Marrow Transplant 11: 688-697, 2005.

10. Zhou SY, Shi YK, He XH, et al: Treatment effect of DICE regimen on patients with relapsed or refractory intermediate and highgrade non-Hodgkin's lymphoma. Ai Zheng 24: 465-469, 2005

11. Clavio M, Garrone A, Pierri I, et al: Ifosfamide, epirubicin, etoposide (IEV) and autologous peripheral blood progenitor cell transplant: A feasible and effective salvage treatment for lymphoid malignancies. Oncol Rep 14: 933-940, 2005.

12. Menzel H, Müller A, von Schilling C, Licht T, Peschel C and Keller U: Ifosfamide, epirubicin and etoposide rituximab in refractory or relapsed B-cell lymphoma: analysis of remission induction and stem cell mobilization. Leuk Lymphoma 49: 1337-1344, 2008.

13. Bishton MJ, Lush RJ, Byrne JL, Russell NH, Shaw BE and Haynes AP: Ifosphamide, etoposide and epirubicin is an effective combined salvage and peripheral blood stem cell mobilisation regimen for transplant-eligible patients with non-Hodgkin lymphoma and Hodgkin disease. Br J Haematol 36: 752-761, 2007.

14. Hertzberg MS, Crombie C, Benson W, Taper J, Gottlieb D and Bradstock KF: Outpatient fractionated ifosfamide, carboplatin, and etoposide as salvage therapy in relapsed and refractory nonHodgkin's lymphoma. Ann Oncol 17 (Suppl 4): 25-30, 2006.

15. Aurer I, Metrovic Z, Nemet D, et al: Treatment of relapsed or refractory aggressive non-Hodgkin lymphoma with two ifosfamide-based regimens, IMVP and ICE. J Chemother 20: 640-644, 2008.

16. Simpson L, Ansell SM, Colgan JP, et al: Effectiveness of second line salvage chemotherapy with ifosfamide, carboplatin and etoposide in patients with relapsed diffuse large B-cell lymphoma not responding to cisplatinum, cytosine arabinoside and dexamethasone. Leuk Lymphoma 48: 1332-1337, 2007.

17. Kewalramani T, Zelenetz AD, Nimer SD, et al: Rituximab and ICE as second-line therapy before autologous stem cell transplantation for relapsed or primary refractory diffuse large B-cell lymphoma. Blood 103: 3684-3688, 2004.

18. Shütt P, Passon J, Ebeling $\mathrm{P}$, et al: Ifosfamide, etoposide, cytarabine and dexamethasone as salvage treatment followed by high-dose cyclophosphamide, melphalan and etoposide with autologous peripheral blood stem cell transplantation for relapsed or refractory lymphomas. Eur J Haematol 78: 93-101, 2007.

19. Müller-Beissenhirtz H, Kasper C, Nückel H and Dührsen U: Gemcitabine, vinorelbine and prednisone for refractory or relapsed aggressive lymphoma, result of a phase II single center study. Ann Hematol 84: 796-801, 2005.

20. Papageorgiou ES, Tsirigotis P, Dimopoulos M, et al: Combination chemotherapy with gemcitabine and vinorelbine in the treatment of relapsed or refractory diffuse large B-cell lymphoma: a phase-II trial by the Hellenic Cooperative Oncology Group. Eur J Haematol 75: 124-129, 2005

21. Avilès A, Neri N, Huerta-Guzman $J$ and Fernandez $R$ : Gemcitabine and cisplatin in refractory malignant lymphoma. Oncology 66: 197-200, 2004.

22. Crump M, Baetz T, Couban S, et al: Gemcitabine, dexamethasone and cisplatin in patients with recurrent or refractory aggressive histology B-cell non-Hodgkin lymphoma. Cancer 101: $1835-1842,2004$

23. Fan Y, Huang ZY, Luo LH and Yu HF: Efficacy of GDP regimen (gemcitabine, dexamethasone and cisplatin) on relapsed or refractory aggressive non-Hodgkin's lymphoma: a report of 24 cases. Ai Zheng 27: 1222-1225, 2008.
24. Di Renzo N, Brugnatelli M, Montanini A, et al: Vinorelbine, gemcitabine, procarbazine and prednisone (ViGePP) as salvage therapy in relapsed or refractorry aggressive non-Hodgkin's lymphoma: results of a phase II study conducted by the Gruppo Italiano per lo Studio dei Linfomi. Leuk Lymphoma 47: 473-479, 2006

25. Corazzelli G, Russo F, Capobianco G, Marcacci G, Della Cioppa P and Pinto A: Gemcitabine, ifosfamide, oxaliplatin and rituximab (R-GIFOX), a new effective cytoreductive/ mobilizing salvage regimen for relapsed and refractory aggressive non-Hodgkin's lymphoma: results of a pilot study. Ann Oncol 17 (Suppl 4): 18-24, 2006.

26. Lòpez A, Gutierrez A, Palacios A, et al: GEMOX-R regimen is a highly effective salvage regimen in patients with refractory/ relapsing diffuse large-cell lymphoma: a phase II study. Eur J Haematol 80: 127-132, 2007.

27. Ng M, Waters J, Cunnigham D, et al: Gemcitabine, cisplatin and methylprednisolone (GEM-P) is an effective salvage regimen in patients with relapsed and refractory lymphoma. Br J Cancer 92: 1352-1357, 2005.

28. Sirohi B, Cunningham D, Norman A, et al: Gemcitabine, cisplatin and methylprednisolone (GEM-P) with or without rituximab in relapsed and refractory patients with diffuse large B-cell lymphoma. Hematology 12: 149-153, 2007.

29. Oki Y, Pro B, Fayad LE, et al: Phase 2 study of gemcitabine in combination with rituximab in patients with recurrent or refractory Hodgkin lymphoma. Cancer 112: 831-836, 2008.

30. Olivieri A, Brunori M, Capelli D, et al: Salvage therapy with an outpatient DHAP schedule followed by PBSC transplantation in 79 lymphoma patients: an intention to mobilize and transplant analysis. Eur J Haematol 72: 10-17, 2004.

31. Mey UJ, Orlopp KS, Flieger D, et al: Dexamethasone, high-dose cytarabine, and cisplatin in combination with rituximab as salvage treatment for patients with relapsed or refractory aggressive non-Hodgkin's lymphoma. Cancer Invest 24: 593-600, 2006.

32. Josting A, Sieniawski M, Glossmann JP, et al: High-dose sequential chemotherapy followed by autologous stem cell transplantation in relapsed or refractory aggressive non-Hodgkin's lymphoma: results of a multicentric phase II study. Ann Oncol 16: 1359-1365, 2005

33. Vellenga E, van Putten WL, van't Veer MB, et al: Rituximab improves the treatment results of DHAP-VIM-DHAP and ASCT in relapsed/progressive aggressive CD20+ $\mathrm{NHL}$ : a prospective randomized HOVON trial. Blood 111: 537-543, 2008.

34. Park SH, Kim S, Ko OB, et al: ESHAP salvage therapy for refractory and relapsed non-Hodgkin's lymphoma: a single center experience. Korean J Intern Med 21: 159-164, 2006.

35. Martin A, Conde E, Arnan M, et al: R-ESHAP as salvage therapy for patients with relapsed or refractory diffuse lsrge B-cell lymphoma: the influence of prior exposure to rituximab on outcome. Haematologica 93: 1829-1836, 2008.

36. Sorà F, Piccirillo N, Chiusolo $\mathrm{P}$, et al: Mitoxantrone, carboplatin, cytosine arabinoside, and methylprednisolone followed by autologous peripheral blood stem cell transplantation. Cancer 106: 859-866, 2006.

37. Atta J, Chow KU, Weidmann E, Mitrou PS, Hoelzer D and Martin H: Dexa-BEAM as salvage therapy in patients with primary refractory aggressive non-Hodgkin lymphoma. Leuk Lymphoma 48: 349-356, 2007.

38. Jermann M, Jost LM, Taverna CH, et al: Rituximab-EPOCH, an effective salvage therapy for relapsed, refractory or transformed B-cell lymphomas: results of a phase II study. Ann Oncol 15: 511-516, 2004.

39. Wang M, Fayad L, Cabanillas F, et al: Phase-2 trial of rituximab plus HyperCVAD alternating with rituximab plus methotrexate - cytarabine for relapsed or refractory aggressive mantle cell lymphoma. Cancer 113: 2734-2741, 2008.

40. Robak T, Lech-Maranda E, Janus A, Blonski J, Wierzbowska A and Gora-Tybor J: Cladribine combined with cyclophosphamide and mitoxantrone is an active salvage therapy in advanced nonHodgkin's lymphoma. Leuk Lymphoma 48: 1092-1101, 2007.

41. Zwick C, Birkmann J, Peter N, et al: Equitoxicity of bolus and infusional etoposide: results of a multi center randomised trial of the German High-Grade Non-Hodgkin Lymphoma Study Group (DSHNHL) in elderly patients with refractory or relapsing aggressive non-Hodgkin lymphoma using the CEMP regimen (cisplatinum, etoposide, mitoxantrone and prednisone). Ann Hematol 87: 717-726, 2008.

42. Sud R and Friedberg JW: Salvage therapy for relapsed or refractory diffuse large B-cell lymphoma: impact of prior rituximab. Haematologica 93: 1776-1780, 2008. 